

\title{
TERMOGRAFÍA INFRARROJA PARA EL DIAGNÓSTICO TÉRMICO CONFIABLE CON ALTA REPLICABILIDAD Y BAJO COSTO DE VIVIENDAS EN MENDOZA, ARGENTINA
}

\author{
THE NATURAL GAS AND ELECTRIC ENERGY CONSUMPTION OF \\ SCHOOL BUILDINGS IN THE METROPOLITAN AREA OF SAN JUAN, \\ ARGENTINA: A STATISTICAL ANALYSIS BASED ON ARCHITECTURAL \\ VARIABLES.
}

\author{
CAROLINA GANEM KARLEN \\ Doctora por la ETSAB -UPC, Arquitecta por la FAU - UM \\ Instituto de Ambiente, Hábitat y Energía INAHE - CONICET / Proyectos \\ de Carreras de Diseño. FAD - UNCUyo. Mendoza, Argentina \\ https://orcid.org/0000-0002-1431-1219 \\ cganem@mendoza-conicet.gov.a
}

\section{RESUMEN}

El objetivo de este trabajo es valorar la utilidad de la termografía infrarroja para diagnosticar en forma rápida el desempeño térmico de edificios existentes. Se seleccionó, para ello, un caso de estudio representativo de vivienda en la ciudad de Mendoza ( $\left.32^{\circ} 40^{\prime} \mathrm{LS}, 68^{\circ} 51^{\prime} \mathrm{LO}, 750 \mathrm{msnm}\right)$ y se procedió a la toma de imágenes termográficas con una cámara de bajo costo (FLIR i3), en dos momentos del día: en la tarde-noche (8 pm) y en la mañana, temprano, (7am), de modo de evitar posibles errores debidos a la incidencia de radiación solar directa. La emisividad se introdujo en la cámara de acuerdo con estudios de materiales locales. Al mismo tiempo, se realizaron mediciones continuas de temperatura del aire dentro y fuera de la vivienda con micro-adquisidores, con el objeto de contrastar los resultados obtenidos mediante la exploración termográfica. Dado que el caso de estudio tiene una inercia térmica alta, las imágenes tomadas durante la tarde-noche muestran en una mayor medida los distintos comportamientos de los materiales de construcción. Se concluye que la termografía infrarroja resulta una herramienta valiosa para un diagnóstico térmico rápido en la certificación edilicia, si se sigue un protocolo específico que permita obtener resultados confiables.

Palabras clave

diagnóstico, temperatura, viviendas apareadas

\section{ABSTRACT}

The aim of the paper is to assess the usefulness of infrared thermography for a quick diagnostic of the thermal performance of the existing building stock. A representative housing case study in the city of Mendoza $\left(32^{\circ} 40^{\prime} \mathrm{SL}\right.$, $68^{\circ} 51^{\prime} \mathrm{WL}, 750 \mathrm{~m}$ above sea level) was selected and infrared imaging was carried out with a low cost thermograph camera (FLIR i3) at two times every day, in the late evening $(8 \mathrm{pm})$ and in the early morning $(7 \mathrm{am})$, for avoiding possible mistakes due to incident direct solar radiation. Emissivity was introduced in the camera according with local material studies. At the same time continuous measurement of air temperature inside and outside were taken with data-loggers with the objective of examining in detail the obtained results by the thermographic survey. Because the case studied has a high thermal inertia the survey in the evening shows better the different behaviour of construction materials. It is concluded that the thermogaphic survey resulted a valuable tool for quick thermal diagnostic in building certification if a specific protocol is followed in order to obtain accurate results.

Keywords 


\section{INTRODUCCIÓN}

La Agencia Internacional de Energía (International Energy Agency - IEA) recomendó a los líderes del G8 una serie de medidas de eficiencia energética en los siguientes aspectos: edificios, equipamiento, iluminación, transporte e industria. Estas consideraciones persiguen logros muy ambiciosos de eficiencia energética que pueden derivar en importantes reducciones de $\mathrm{CO}_{2}$. La IEA estima que, si estas medidas se aplican a escala global, las reducciones estarán entre 4400 y $6800 \mathrm{MtCO}_{2}$ /año para 2030. Esta cantidad equivale lo que fue el total de las emisiones de Estados Unidos de América en el año 2004. Las medidas relacionadas con los edificios incluyen: normativa, monitoreo y evaluación y se focalizan en tres aspectos principales: 1. Códigos edilicios para nuevos edificios; 2 . Viviendas pasivas y edificios energéticamente eficientes; y, 3. Edificios existentes (Schneider, 2015).

Para reducir el consumo energético en edificios existentes en forma masiva, se necesitan técnicas confiables y rápidas de diagnóstico. El monitoreo tradicional del comportamiento térmico de espacios en edificios con micro-adquisidores y termocuplas, como el descripto por Andersen et al., (2017) y Andreoni Trentacoste y Ganem Karlen (2017), consume tiempo y presenta limitaciones en relación con el instrumental y la cantidad de personal involucrado cuando se piensa en una implementación masiva.

La termografía infrarroja es una tecnología de investigación no destructiva y se ha utilizado durante los últimos 30 años para probar y diagnosticar edificios. Resultó ser un método efectivo, conveniente y económico cuando se busca detectar características ocultas de estructuras de edificios (formas preexistentes, cambios estructurales, anomalías estructurales, presencia de cavidades), evaluar HVAC (sistema de calefacción, ventilación y aire acondicionado), detectar degradación (grietas), identificar fuentes de fuga de aire, determinar pérdidas de calor, mapear humedad, verificar aislamiento y evaluar tratamientos de conservación (Kylili et al., 2014; Fox et al., 2015; Fox, Goodhew y De Wilde, 2016; Lucchi, 2018; Kirimtat y Krejcar, 2018; Rocha, Santos y Póvoas, 2018).

En este trabajo se presenta como el primer paso para complementar el monitoreo tradicional. Muchos investigadores han usado esta técnica de forma efectiva con diferentes objetivos, demostrando su gran potencial. Ganem, Barea y Balter (2016) han descrito a la termografía infrarroja como una herramienta valiosa para la inspección y la ejecución de pruebas no-destructivas en elementos edilicios. Y la Organización Internacional de Estándares está trabajando en la ISO/TC 135/SC serie 8, que trata sobre termografía infrarroja en evaluaciones no-destructivas, de las cuales la única norma publicada es la ISO 10878:2013.

Además, Porras-Amores, Mazarrón y Cañas (2013) presentan una metodología que plantea utilizar la termografía cuantitativa infrarroja para determinar la temperatura interior del aire. Y Tejedor et al. (2017) y Nardi et al. (2018) han aplicado la termografía infrarroja con el fin de definir el coeficiente de transferencia global o U-Value en las envolventes edilicias.

Martín Ocaña, Cañas Guerrero y González Requena (2004), por su parte, han propuesto un protocolo basado en la norma BS EN 13187:1999 para evitar errores comunes en las mediciones con termografía causados por la radiación solar incidente. Flores Larsen y Hongn (2012) reportan diferencias entre $25^{\circ} \mathrm{C}$ y $50^{\circ} \mathrm{C}$ cuando se toman imágenes termográficas del mismo elemento constructivo, en un mismo momento, pero con distintos valores de emisividad: 0.9 (valor probable muy cercano al real) y 0.1 (valor muy alejado del real). Al respecto, concluyen que es importante destacar que, en el caso de aplicaciones cualitativas, el uso de una u otra emisividad no afecta más que a un cambio de escalas para ajustar los colores de la imagen. En cambio, en una aplicación cuantitativa en donde el objetivo es conocer la temperatura de la superficie, es fundamental que la emisividad con que se toma el termograma sea cercana al valor real. Asimismo, De Prada Pérez de Azpeitia (2016) y Costilla Sanz (2017) afirman que las consideraciones de emisividad en la termografía son claves para la certeza de la información obtenida. El comportamiento térmico de los materiales está determinado principalmente por sus propiedades ópticas y térmicas. El albedo y la emisividad térmica son los dos factores principales.

A partir de los antecedentes expuestos, el objetivo de este trabajo es evaluar la utilidad de la termografía infrarroja para un diagnóstico rápido y confiable del comportamiento térmico del conjunto de los edificios existentes. Este diagnóstico pretende detectar los aspectos principales y otorgar criterios que orienten inspecciones sucesivas de monitoreo con micro-adquisidores.

\section{METODOLOGÍA}

La termografía infrarroja es una técnica que transforma el patrón de emisiones de un objeto en una imagen visible. Todas las cámaras interpretan la radiación infrarroja proveniente de la superficie investigada, que involucra radiación emitida, reflejada y ocasionalmente transmitida.

El principio de funcionamiento de la termografía infrarroja coincide con las tres leyes esenciales de la radiación:

1. La Ley de Kirchhoff de radiación térmica, que establece la relación entre la emisión y la absorción de energía. Indica que un cuerpo que absorbe mucho también emite mucho $y$, de acuerdo con este principio, el coeficiente de emisión, e, se introduce en la ecuación como la relación de la emisividad, E, del cuerpo real a la emisividad, $E_{z^{\prime}}$ del cuerpo negro bajo la misma temperatura $e=E / E_{z}$. El coeficiente de emisión es nodimensional y tiene valores entre 0 y 1 , dependiendo de la longitud de onda, de la temperatura y de la textura del cuerpo. 
La Ley de Planck de radiación, describe la radiación espectral específica l' que emana el cuerpo negro ideal. Ecuación 1 (Mohr, Taylor y Newell, 2008).

$$
\mathrm{I}^{\prime}(\lambda, \mathrm{T})=\frac{2 \pi \mathrm{hc}^{2}}{\lambda^{5}} \cdot \frac{1}{\mathrm{e}^{\mathrm{hc} / \lambda \mathrm{kT}}-1}
$$

Donde:

I es la longitud de onda

$\mathrm{T}$ es la temperatura absoluta $\mathrm{h}$ es la constante de Planck

c es la velocidad de la luz

e es la base del logaritmo natural 2,718281 k es la constante de Boltzmann

Si la radiación espectral específica, I, se grafica en la longitud de onda, l, en función de la temperatura, se obtiene como resultado las curvas típicas de Planck. En ellas se puede observar que el máximo de las curvas cambia con el incremento de la temperatura hacia una longitud de onda menor de acuerdo con la Ley de desplazamiento de Wien. Ecuación 2 (ibidem).

$$
\lambda=\mathrm{b} / \mathrm{T}
$$

Donde:

I es el largo de onda máximo,

T es la temperatura absoluta del cuerpo negro,

b es un constante de proporcionalidad llamado constante de desplazamiento de Wien equivalente a $2.8977685(51) \times 10^{-3} \mathrm{~m} \cdot \mathrm{K}$

La Ley de Stefan-Boltzmann, aplicada a la emisión de una superficie en todas las longitudes de onda, integra la Ley de Planck. El poder radiante I, [W/m²], se incrementa con el cuarto poder de la temperatura. Ecuación 3 (Duffie y Beckmann, 1991).

$$
E=\varepsilon \sigma \mathrm{T}^{4}
$$

\section{Donde:}

E es la energía de la radiación $\left(\mathrm{W} / \mathrm{m}^{2}\right)$,

e es la emisividad hemisférica total de la superficie

$(0<\mathrm{e}<1)$ (no-dimensional), es la constante de Stefan-Boltzmann (5.67051 x $10^{-8} \mathrm{~W} / \mathrm{m}^{2} \mathrm{~K}^{4}$ )

$\mathrm{T}$ es la temperatura (K).

Para este estudio se utilizó una cámara infrarroja marca FLIR, modelo i3, de bajo costo, fácil de usar, pequeña y liviana. Este tipo de cámara es factible que sea empleada, por ejemplo, por técnicos municipales, para realizar diagnósticos generales del conjunto de edificios existentes. Sus especificaciones técnicas son (FLIR, 2018):

$\begin{array}{ll}\text { - } & \text { Resolución: } 60 \times 60 \text { pixeles. } \\ \text { - } & \text { Pixeles totales: } 3,600 \text { pixeles. } \\ \text { - } & \text { Campo de visión: } 12.5^{\circ} \times 12.5^{\circ} \\ \text { - } & \text { Sensibilidad térmica: }<0.15^{\circ} \mathrm{C} . \\ \text { - } & \text { Precisión: }+/-2 \% \circ 2^{\circ} \mathrm{C} . \\ \text { - } & \text { Rango de temperatura: }-20^{\circ} \mathrm{C} \text { a } 250^{\circ} \mathrm{C} . \\ \text { - } & \text { Emisividad: } 0.1 \text { a } 1.0 \text { Ajustable. }\end{array}$

Debido a que existen muy pocos trabajos publicados que informen valores de emisividad de materiales de construcción, es importante realizar pruebas de laboratorio dentro de un rango de temperatura preestablecido. En el presente trabajo, los valores de emisividad fueron introducidos en la cámara de acuerdo a lo informado en estudios locales sobre las propiedades ópticas de los materiales (Alchapar y Correa, 2015). Estos valores son coincidentes con los de Martín Ocaña, Cañas Guerrero y González Requena (2004) que expresan que: la mayoría de los materiales de construcción (especialmente los tradicionales) tienen valores de emisividad entre 0.9 y 0.95 ; estos valores elevados hacen posible la obtención de valores de temperatura certeros mediante la termografía.

Paralelamente, se realizaron mediciones continuas dentro y fuera del edificio cada 15 minutos con microadquisidores LASCAR EL-USB-TC-LCD, con el propósito de examinar en detalle los resultados obtenidos mediante la auditoría termográfica. Los micro-adquisidores se ubicaron de acuerdo con las siguientes premisas:

- Los micro-adquisidores deben ubicarse suspendidos en el espacio y alejados de estructuras con masa térmica. Si se encuentran en el exterior, deben estar protegidos de la radiación directa.

- La distancia del suelo se fijó en 2 metros en todos los casos.

- Las mediciones deben tomarse cada 15 minutos por el lapso continuo de una semana o más. En este caso se realizaron mediciones por 1 mes.

- $\quad$ Antes de ser colocados en el edificio, los micro-adquisidores deben programarse para iniciar al mismo tiempo y mantenerse juntos midiendo por un determinado tiempo en las mismas condiciones, a modo de evaluación. De esta forma, se podrá detectar si hay diferencia entre los sensores.

Las mediciones y las imágenes termográficas se tomaron en la estación de verano para el Hemisferio Sur, desde el 19 de enero al 21 de febrero de 2016, en un caso representativo de la ciudad de Mendoza, Argentina ( $\left.32^{\circ} 40^{\prime} \mathrm{LS}, 68^{\circ} 51^{\prime} \mathrm{LO}, 750 \mathrm{msnm}\right)$. Mendoza tiene un clima templado continental seco, con importantes 
variaciones de temperatura diaria y estacional. De acuerdo con la clasificación de Geiger y Pohl (1953), Mendoza tiene un clima Bwk. En la Tabla 1, se presenta información climática relativa a la temperatura y a la radiación solar.

\begin{tabular}{|l|l|}
\hline Valores anuales & \\
\hline Temperatura máxima media & $24.5^{\circ} \mathrm{C}$ \\
Temperatura mínima media & $9.6^{\circ} \mathrm{C}$ \\
Temperatura media & $16.5^{\circ} \mathrm{C}$ \\
Irradiancia global horizontal & $18.4 \mathrm{MJ} / \mathrm{m}^{2}$ \\
Humedad relativa & $56 \%$ \\
Heliofanía relativa & $63 \%$ \\
\hline Julio & \\
\hline Temperatura máxima media & $15.7^{\circ} \mathrm{C}$ \\
Temperatura mínima media & $0.8^{\circ} \mathrm{C}$ \\
Temperatura media & $7.3^{\circ} \mathrm{C}$ \\
Irradiancia global horizontal & $10.2 \mathrm{MJ} / \mathrm{m}^{2}$ \\
Humedad relativa & $63 \%$ \\
Heliofanía relativa & $58 \%$ \\
\hline Enero & $24.9^{\circ} \mathrm{C}$ \\
\hline Temperatura máxima media & $26.1 \mathrm{MJ} / \mathrm{m}^{2}$ \\
Temperatura mínima media & $49 \%$ \\
Temperatura media & $66 \%$ \\
Irradiancia global horizontal & $32.3^{\circ} \mathrm{C}$ \\
Humedad relativa & $17.4^{\circ} \mathrm{C}$ \\
Heliofanía relativa & $215^{\circ} \mathrm{C}$ \\
\hline Grados día calefacción (Tb $\left.=18^{\circ} \mathrm{C}\right)$ & \\
Grados día refrigeración (Tb $\left.=23^{\circ} \mathrm{C}\right)$ & $\mathrm{C}$ \\
\hline
\end{tabular}

Tabla 1. Información climática para Mendoza, Argentina. Fuente: Servicio Meteorológico Nacional - Fuerza Aérea Argentina.

\section{CASO DE ESTUDIO}

De acuerdo con el último Censo Nacional de Población, Hogares y Viviendas (INDEC, 2010) la Provincia de Mendoza cuenta con un total de 539.271 viviendas, de las cuales 538.056 son particulares y 1.215 , colectivas. Como primera aproximación a la selección del caso de estudio, se analizaron 16 perfiles urbanos en el Barrio Bombal Sur, en la ciudad de Mendoza. Este barrio se caracteriza por estar consolidado y por ser representativo del tipo de vivienda unifamiliar de baja altura con colindancia, característico de la edificación predominante en la ciudad.
En la Figura 1, se puede observar una imagen satelital y un plano de un sector de la trama consolidada de la ciudad de Mendoza. En ellas, se identifican con color los límites del Barrio Bombal Sur entre las calles Hipólito Irigoyen al Norte, Coronel Beltrán hacia el Oeste, Anzorena hacia el Sur y la Avenida General San Martín hacia el Este. Asimismo, se localiza el caso de estudio y se presenta una vista de la calle en donde se puede apreciar, a la derecha, la fachada Norte de la vivienda identificada con el número 32.

Se tomaron imágenes termográficas acompañadas por imágenes fotográficas para observar la diversidad de comportamientos de las diferentes viviendas en los dos momentos pautados para evitar la incidencia de la radiación solar directa: a la mañana, temprano, y a la tardenoche. En la Figura 2 se muestran algunos ejemplos del relevamiento efectuado.

A partir del examen de los primeros registros, se seleccionó una tipología de vivienda para la aplicación del diagnóstico térmico rápido en conjunto con las mediciones de temperatura con micro- adquisidores: el chalet neo-colonial. Esta tipología es muy habitual en las ciudades argentinas y en otros países latinoamericanos que recibieron inmigración española e italiana. Se trata de una tipología mediterránea que se construye desde 1940 y es todavía el tipo de vivienda más elegido de la clase media.

La vivienda seleccionada, ubicada en la calle Tucumán, número 32, fue construida en 1960. Es una vivienda de dos plantas, semi-compacta entre medianeras. Su fachada principal se orienta al Ecuador (es decir, al Norte en el Hemisferio Sur). La Figura 1 (c) muestra una fotografía general de la calle Tucumán entre la numeración 0 y 50 , en cuya derecha se puede notar la fachada norte de la vivienda identificada con el número 32.

Las paredes son de $0.22 \mathrm{~m}$ de espesor, fabricadas con ladrillo macizo, cuyas dimensiones son: $0.27 \times 0.17 \times$ $0.7 \mathrm{~m}$ y peso promedio, de $3,51 \mathrm{Kg} / \mathrm{unidad}$. Este material es usualmente conocido con el nombre de "ladrillón" por sus dimensiones mayores a las estándar, las que permiten construir paredes antisísmicas. Las paredes están recubiertas con revoque grueso y luego con revoque fino, pintadas en un color beige claro, sin aislamiento térmico $\left(\mathrm{U}=2.4 \mathrm{~W} / \mathrm{m}^{2} \mathrm{~K}\right.$, este valor corresponde a la suma de las capas que se describen).

Los techos tienen estructura de madera con machimbre, aislamiento de $0.05 \mathrm{~m}$ de poliestireno expandido, una capa de hormigón pobre y, sobre esta tejas, cerámicas rojas de tipo español. Los techos están inclinados $25^{\circ}$, siguiendo el eje Este-Oeste. Los espacios interiores tienen cielo raso suspendido de yeso $\left(\mathrm{U}=1.1 \mathrm{~W} / \mathrm{m}^{2} \mathrm{~K}\right.$, este valor corresponde al sistema compuesto por las distintas capas que se describen).

El $20 \%$ de la fachada Norte está cubierto por ventanas. Estas presentan marcos de madera en color blanco y 

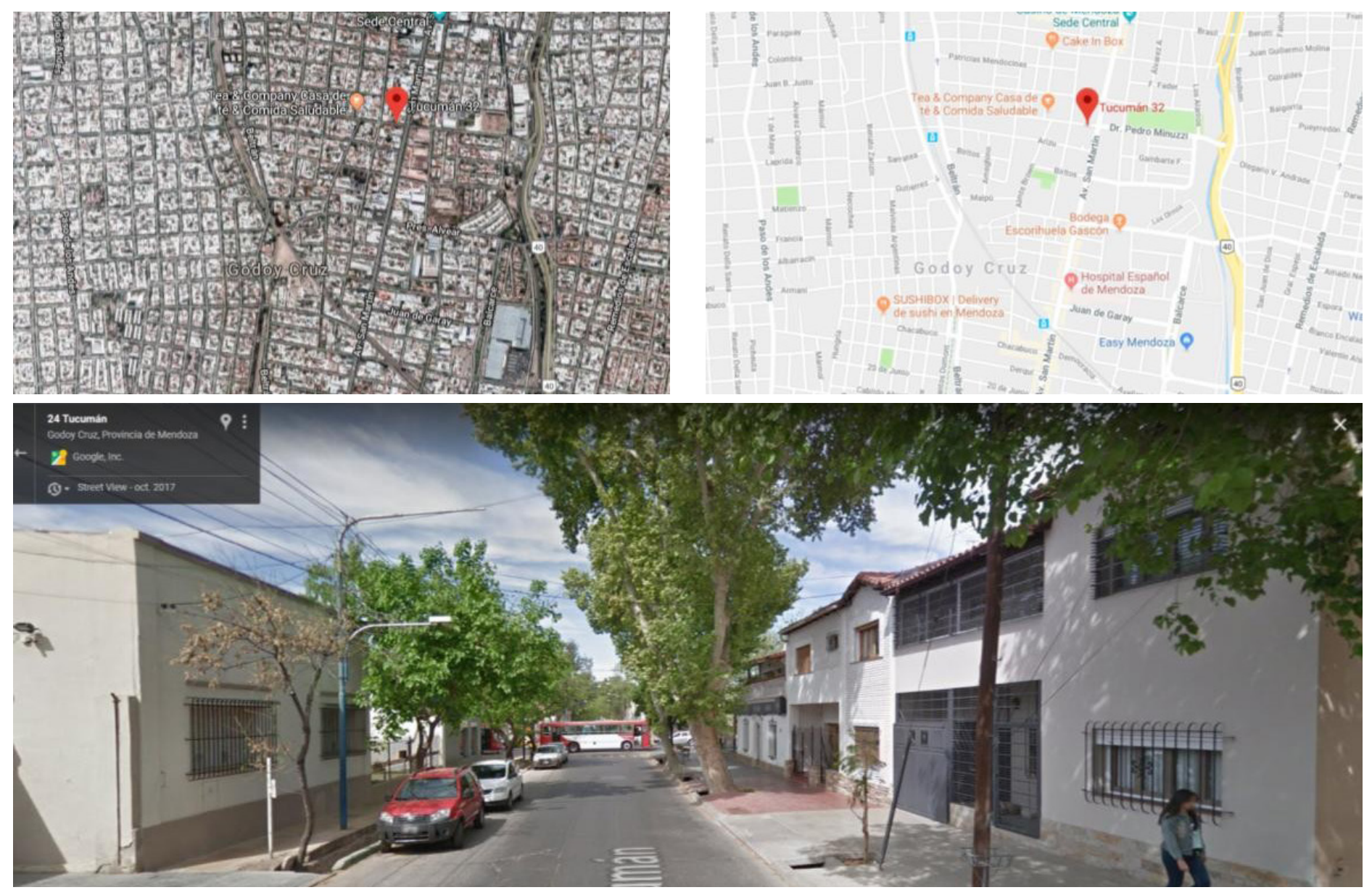

Figura 1. De arriba hacia abajo y de izquierda a derecha: (a) Imagen satelital parcial de la ciudad de Mendoza, ubicación del Barrio Bombal Sur y de caso de estudio; (b) Plano parcial de la ciudad de Mendoza, y (c) Vista de la calle Tucumán entre la numeración 0 y 50 . A la derecha, con la fachada orientada al Norte, se encuentra la vivienda identificada con el número 32. Fuente: Google Maps. [Consultado noviembre 2018].

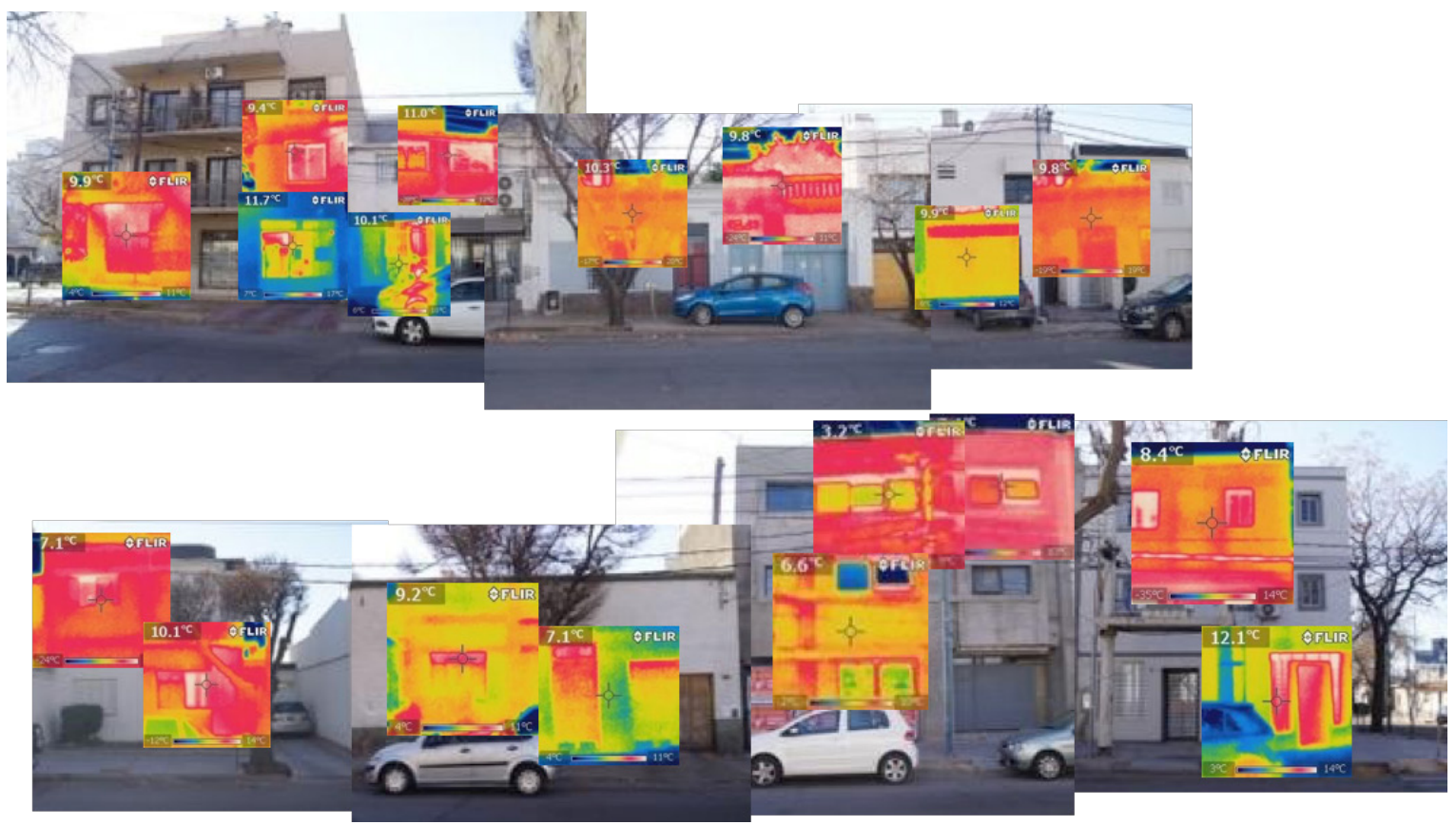


simple vidrio. Las ventanas están protegidas en el interior por cortinas y en el exterior por cortinas de enrollar de madera pintadas en color blanco $\left(U=3.1 \mathrm{~W} / \mathrm{m}^{2} \mathrm{~K}\right.$, este valor corresponde al sistema de ventanas con protecciones).

\section{RESULTADOS Y DISCUSIÓN}

La Figura 3 exhibe las mediciones tomadas durante el 3 y 4 de febrero. Tales días fueron seleccionados para analizar las imágenes termográficas en este estudio porque son los que más se aproximan al día promedio de la estación y porque están rodeados por días similares, lo cual proporciona estabilidad al comportamiento térmico de la vivienda. Las imágenes termográficas se tomaron a las 7 am, horario previo a la salida del sol, y a las 8 pm, horario posterior a la puesta del sol.

En el periodo seleccionado, las temperaturas interiores presentaron variaciones de $1^{\circ} \mathrm{C}$, registrándose entre los $27^{\circ} \mathrm{C}$ y los $28^{\circ} \mathrm{C}$. Y, las temperaturas exteriores variaron diariamente $10^{\circ} \mathrm{C}$, desde $20.5^{\circ} \mathrm{C}$ a $30.5^{\circ} \mathrm{C}$. Aunque hay un potencial excepcional para utilizar la estrategia de ventilación nocturna para enfriar espacios interiores, las ventanas se mantienen cerradas la mayoría del día.

En la mañana, al momento de la realización de la inspección termográfica, a las 7 am, la temperatura dentro de la casa era de $27^{\circ} \mathrm{C}$ y la exterior, de $24^{\circ} \mathrm{C}$. El gradiente térmico era de $3^{\circ} \mathrm{C}$. En la tarde- noche, al momento de la realización de la inspección termográfica, a las 8pm, la temperatura dentro de la vivienda era de $28^{\circ} \mathrm{C}$ y la temperatura exterior, de $25^{\circ} \mathrm{C}$. El gradiente de temperatura se mantuvo en $3^{\circ} \mathrm{C}$. Este resultado es consistente con las investigaciones de Martín Ocaña, Cañas Guerrero y González Requena (2004), en las que se expresa que los edificios macizos, construidos en su envolvente con materiales con alta densidad, presentan gradientes de $3^{\circ} \mathrm{C}$ entre las temperaturas interiores y las exteriores.

La Figura 4 muestra, de izquierda a derecha, una fotografía de la fachada Norte desde el espacio interior, la imagen termográfica registrada temprano por la mañana, a las 7 am, y la imagen termográfica registrada a la tarde-noche, a las 8 pm.

La escala de temperaturas en el registro termográfico efectuado a la mañana temprano varía entre $25^{\circ} \mathrm{C}$ y $31^{\circ} \mathrm{C}$, mientras que en la tarde noche, las temperaturas registradas varían entre $30^{\circ} \mathrm{C}$ y $37^{\circ} \mathrm{C}$. Las temperaturas superficiales oscilan entre $5^{\circ} \mathrm{C}$ y $6^{\circ} \mathrm{C}$, mientras que la temperatura del aire sólo varía $1^{\circ} \mathrm{C}$. La masa del edificio se ha enfriado con las menores temperaturas nocturnas; sin embargo, como las ventanas permanecen cerradas, la temperatura del aire se mantiene.

Se puede observar que, en la mañana temprano, las temperaturas que evidencia la imagen termográfica son casi homogéneas, entre $27^{\circ} \mathrm{C}$ y $28^{\circ} \mathrm{C}$. En el caso de la imagen de la tarde-noche, las temperaturas de cada elemento constructivo varían y es posible identificar con mayor claridad la ubicación de la ventana en la pared. Por ejemplo, se observa cómo el

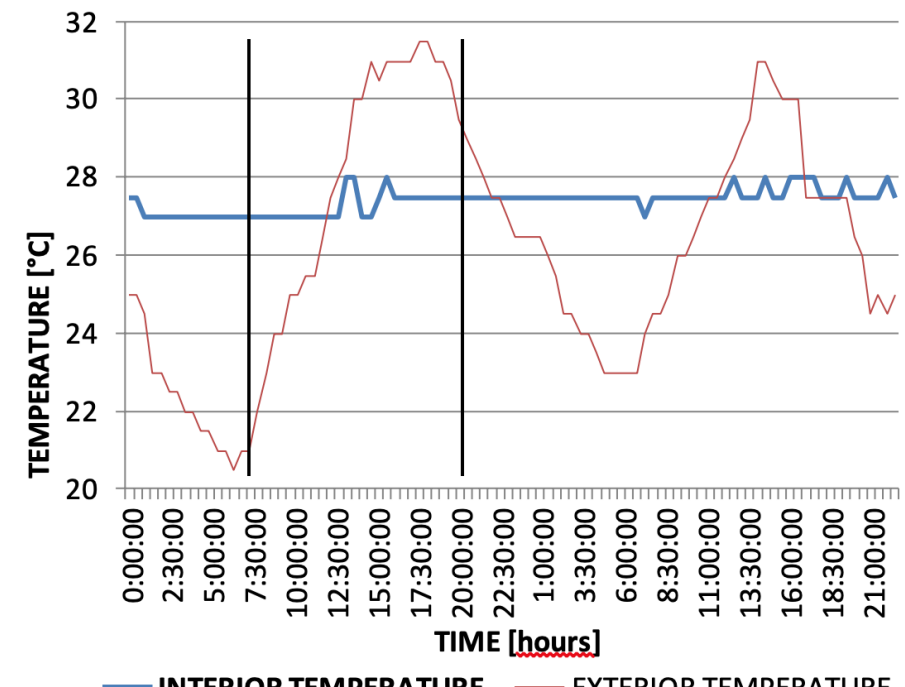

Figura 3. Temperatura interior y exterior de la vivienda durante los días que se realizaron las inspecciones termográficas, a las 7 am y a las 8 pm. Fuente: Elaboración de la autora.
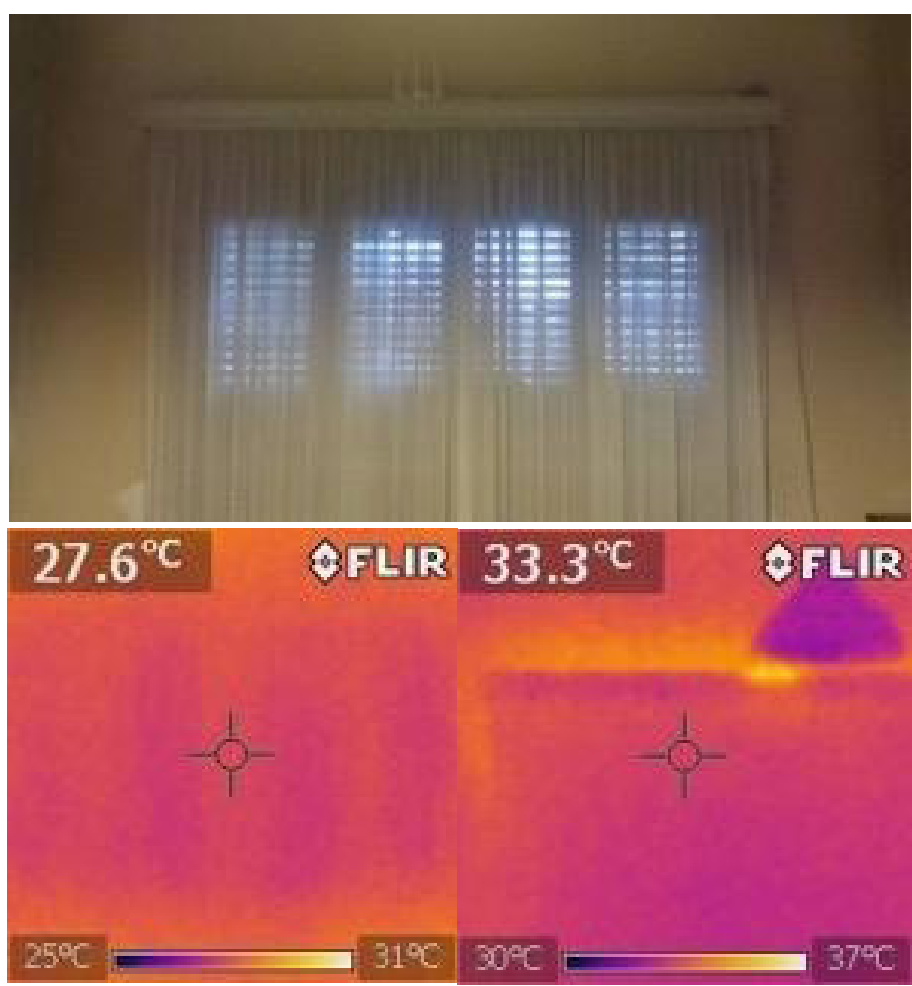

Figura 4. De izquierda a derecha: (a) Fotografía de la pared interor. (b) Imagen termográfica a las 7 am. (c) Imagen termográfica a las 8pm. Fuente: Elaboración de la autora. 

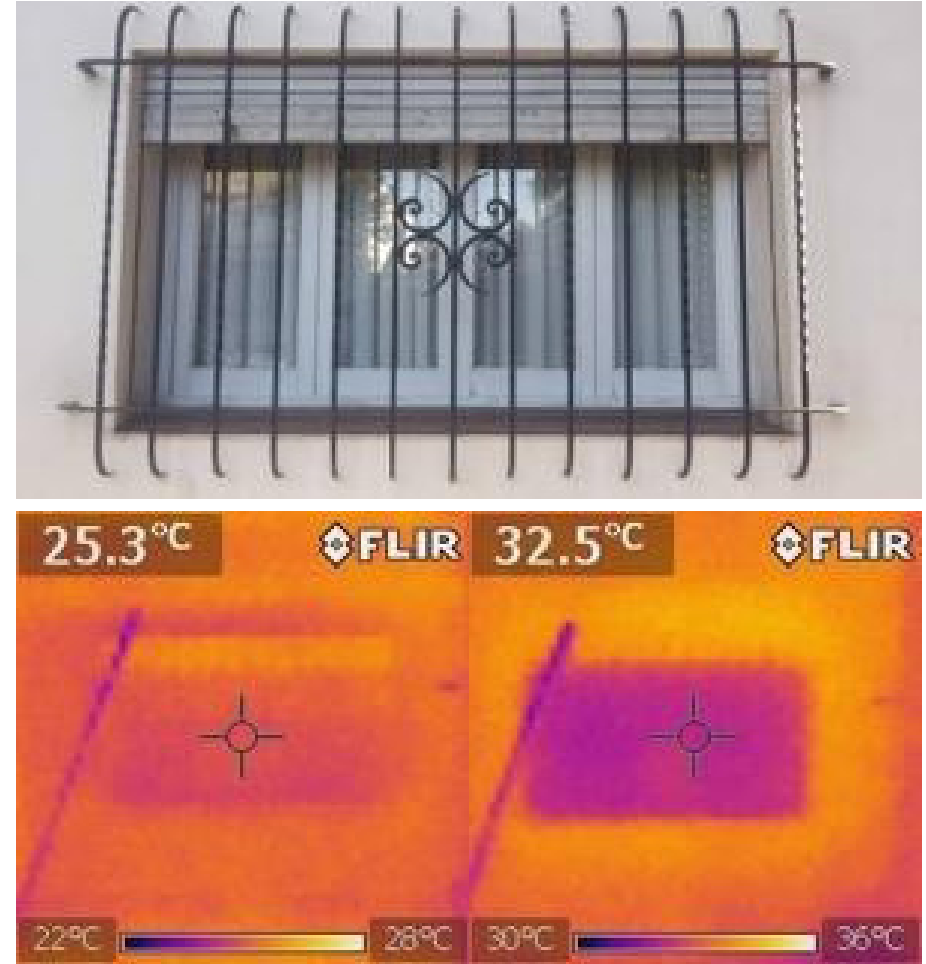

Figura 5. De izquierda a derecha: (a) Fotografía de la fachada Norte desde el exterior. (b) Imagen termográfica, a las 7 am. (c) Imagen termográfica a las 8 pm. Fuente: Elaboración de la autora.
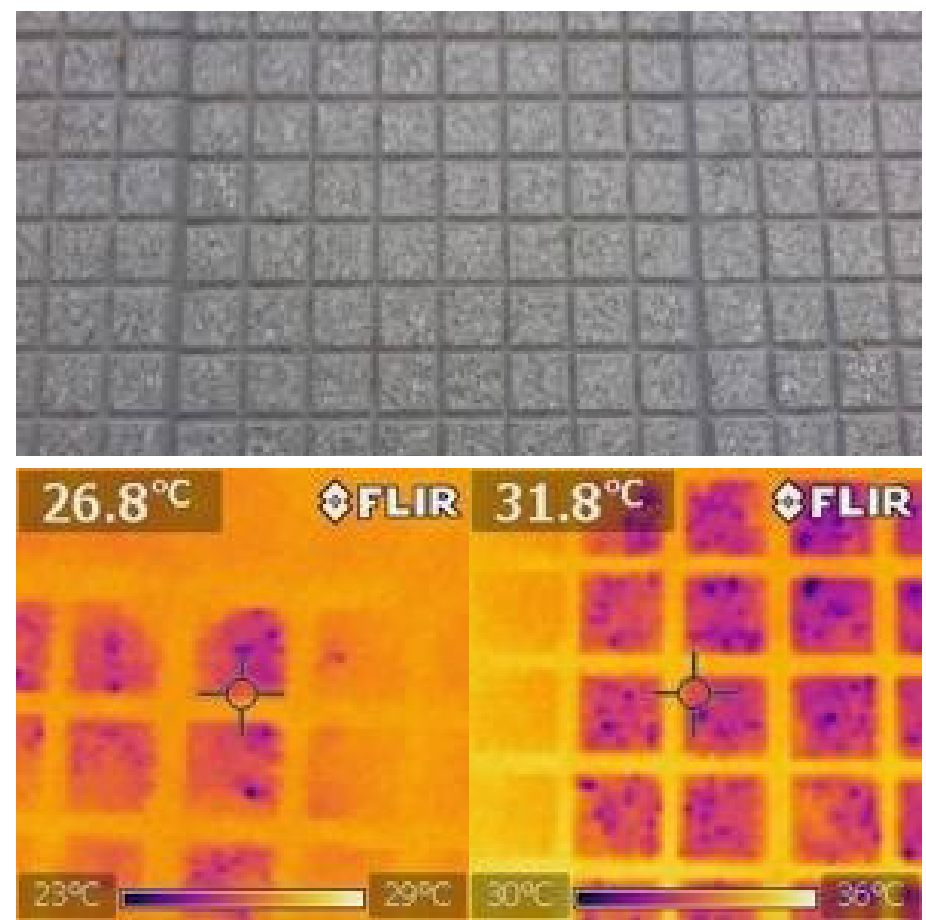

Figura 6. De izquierda a derecha: (a) Fotografía de la vereda. (b) Imagen termográfica a las 7 am. (c) Imagen termográfica a las 8 pm. Fuente: Elaboración de la autora. calor se conserva sobre la estructura superior que contiene a la cortina.

La Figura 5, de izquierda a derecha, muestra una fotografía de la fachada Norte desde el espacio exterior, la imagen termográfica registrada temprano por la mañana y la imagen termográfica registrada a la tarde-noche.

La escala de temperaturas en el registro termográfico efectuado a la mañana temprano varía entre $22^{\circ} \mathrm{C}$ y $28^{\circ} \mathrm{C}$, mientras que en la tarde noche, las temperaturas registradas varían entre $30^{\circ} \mathrm{C}$ y $36^{\circ} \mathrm{C}$. Las temperaturas superficiales varían $8^{\circ} \mathrm{C}$, mientras que la temperatura del aire varía $10^{\circ} \mathrm{C}$. La masa del edificio se ha enfriado con las menores temperaturas nocturnas y las temperaturas superficiales son cercanas a las temperaturas del aire.

En la imagen termográfica tomada en la mañana temprano, una vez más las imágenes son casi homogéneas, mientras que en el caso de la tarde-noche, las diferencias entre los materiales livianos y pesados de la envolvente se observan con claridad. La cortina de enrollar de madera presenta menores temperaturas que la pared de ladrillo macizo.

Nótese que la temperatura superficial en la tarde-noche en el interior de la vivienda (Figura 4 (c)) y la correspondiente al exterior (Figura 5 (c)) se encuentran en el mismo rango entre: $30^{\circ} \mathrm{C}$ y $36 / 37$

${ }^{\circ} \mathrm{C}$.

El mismo comportamiento en la temperatura descripto en las imágenes exteriores en la fachada Norte se puede advertir en la vereda de la vivienda. La Figura 6, de izquierda a derecha, expone una fotografía de la vereda, la imagen termográfica registrada temprano por la mañana y la imagen termográfica registrada a la tarde-noche.

La escala de temperaturas en la mañana se encuentra entre $23^{\circ} \mathrm{C}$ y $29^{\circ} \mathrm{C}$ (un grado centígrado por sobre las temperaturas registradas en la fachada Norte), mientras que en la imagen termográfica de la tarde-noche las temperaturas varían entre $30^{\circ} \mathrm{C}$ y $36^{\circ} \mathrm{C}$, encontrándose en el mismo rango que en la fachada Norte.

La diferencia entre la fachada y la vereda a la mañana temprano se justifica con la cantidad de masa que acumula calor durante el día que, en el caso de la vereda, no alcanza a disiparse de la misma manera que la pared durante la noche, cuando las temperaturas exteriores son más bajas. Por lo tanto, la vereda inicia el día "más caliente" con mayor cantidad de calor acumulado.

\section{CONCLUSIÓN}

Las imágenes tomadas en la mañana temprano tienden a revelar temperaturas homogéneas en las superficies. Debido a que la vivienda en estudio tiene alta inercia térmica, el registro en la tarde-noche muestra mejor 
los diferentes comportamientos de los materiales de construcción.

Si el objetivo del diagnóstico termográfico es evaluar cómo el calor se distribuye en una construcción dada, detectar puentes térmicos u otro tipo de intercambio energético, la tarde-noche será el mejor momento para realizar el registro termográfico.

Asimismo, si se pretende comprender el comportamiento térmico edilicio en relación a las posibilidades de aplicación de la estrategia de ventilación nocturna para el refrescamiento de los espacios interiores en forma natural, se sugiere el registro en la mañana temprano y en la tarde-noche. De esta forma, será posible cuantificar el calor acumulado durante el día en las edificaciones y valorar el enfriamiento de las mismas durante la noche.

Otra aplicación interesante es la posibilidad de determinar el valor $U$ de la envolvente edilicia utilizando una cámara infrarroja. Si el coeficiente de transmisión de calor está determinado, las imágenes termográficas permiten conocer la conductividad de una pared homogénea y la conductividad efectiva de una pared con gran precisión.

Thouvenel (2012) indica: el error en la determinación del valor $U$ de un componente edilicio mediante termografía es menor al $10 \%$. Cuando el coeficiente de transferencia de calor se determina con la cámara, el método que mejor funciona consiste en considerar el punto central de temperatura provisto por la cámara, y utilizar el total del área para el cálculo. El punto central será representativo del total del área.

Tomar el punto central de temperatura para obtener el mejor resultado puede ser lógico, debido a que las fórmulas utilizadas para calcular todos los valores toman la conducción de calor en una pared unidimensional. En el punto central, esta aproximación es la más precisa. El experimento se lleva a cabo normalmente en tres dimensiones ya que el flujo de calor no se genera sólo en la dirección x sino también en otras direcciones.

No obstante esto, el método que se propone debe ser rápido y permitir determinar las propiedades de los materiales, aproximándose a los valores reales, pero no supone obtener resultados exactos. Desde esta perspectiva, la aproximación unidimensional funciona, tomando la temperatura del centro de la pared para obtener resultados confiables.

Se concluye, en definitiva, que el registro termográfico resulta una herramienta valiosa para el diagnóstico térmico rápido para la certificación edilicia, como complemento a la medición intensiva con micro- adquisidores de casos de control. Se debe seguir un protocolo específico que paute cuándo y cómo se obtiene el registro termográfico y el valor de la emisividad que se introducirá previamente en la cámara. Sólo si estos aspectos se tienen en cuenta, es posible la obtención de resultados confiables.

\section{PROSPECTIVA}

A partir de la investigación que se presenta en este artículo, quedan abiertas líneas de trabajo que se continuarán a futuro. Se prevé continuar el relevamiento y diagnóstico de diferentes viviendas dentro de la misma trama consolidada del Barrio Bombal Sur, con las mismas características constructivas y tipológicas a fin de fortalecer el número de casos de aplicación.

Igualmente, se prevé realizar el mismo procedimiento metodológico con viviendas que presenten otro tipo de materialidad, la que, si bien no es representativa de los edificios existentes en la actualidad, constituye una alternativa en el futuro, como es el caso de los materiales livianos con aislamiento térmico.

\section{AGRADECIMIENTOS}

Los autores agradecen el financiamiento recibido del CONICET y del FONCyT, y a los propietarios de la vivienda que permitieron realizar las mediciones térmicas y el registro termográfico.

\section{REFERENCIAS BIBLIOGRÁFICAS}

ALCHAPAR, Noelia y CORREA, Érica. Solar reflectance of opaque enveloped and its effect on urban temperatures, Revista Informes de la Construcción, 2015, vol. 67, n 540, pp.112-124.

ANDERSEN, Micaela; DÍsCOLI, Carlo Alberto; VIEGAS, Graciela Melisa y MARTINI, Irene. Monitoreo energético y estrategias de RETROFIT para viviendas sociales en clima frío, Revista Hábitat Sustentable, 2017, vol.7, n² 2, pp. 50-63.

ANDREONI TRENTACOSTE, Soledad Elisa y GANEM KARLEN, Carolina. Influencia del uso y gestión de la envolvente en el comportamiento térmico de verano de una vivienda en la ciudad de Mendoza, Argentina, Revista Hábitat Sustentable, 2017, vol.7, n², pp. 64-75.

COSTILLA SANZ, Carlos. Aplicación práctica para cámara IR (Infrarrojos). Proyecto fin de carrera. Trabajo fin de grado, E.T.S.I. y

Sistemas de Telecomunicación (UPM), Madrid, 2017.

DE PRADA PÉREZ DE AZPEITIA, Fernando Ignazio. La termografía infrarroja: un sorprendente recurso para la enseñanza de la física y la química, Revista Eureka sobre Enseñanza y Divulgación de las Ciencias, 2016, vol. 13, pp. 617-627

DUFFIE, John A. y BECKMAN, William A. Solar Engineering and Thermal Processes. $2^{\text {da }}$ edición. New York: John Wiley \& Sons, 1991. 
FLIR. Guía sobre termografía para aplicaciones en edificios y energía renovable [en línea]. [Consultado: 12 noviembre 2018] Disponible en: http://www.flirmedia.com/MMC/THG/ Brochures/T820325/T820325_ES.pdf

FLORES LARSEN, Silvana y HONGN, Marcos. Termografía infrarroja en la edificación: aplicaciones cualitativas, Avances en Energías Renovables y Medio Ambiente, 2012, vol. 16, pp. 8.25-8.32.

FOX, Mathew; COLEY, David; GOODHEW, Steve y DE WILDE, Pieter. Time-lapse thermography for building defect detection, Energy and Buildings, 2015, vol. 92, pp. 95-106.

FOX, Mathew; GOODHEW, Steve y DE WILDE, Pieter. Building defect detection: External versus internal thermography, Building and Environment, 2016, vol. 105, pp. 317-331.

GANEM, Carolina; BAREA, Gustavo y BALTER, Julieta. Infrared Thermography for Quick Thermal Diagnostic of Existing Building. En: Proceedings of PLEA 2016 (Los Ángeles, 11-13 de Julio de 2016) Cities, Buildings, People: Towards Regenerative Environments. Los Ángeles, 2016.

GEIGER R. y POHL W. Revision of Koeppen-Geiger climate maps of the Earth. Germany: Justus Perthes, 1953.

INDEC. Censo Nacional de Población, Hogares y Viviendas 2010 [en línea]. [Consultado 8 noviembre 2018]. Disponible en: https://www.indec.gob.ar.

KIRIMTAT, Ayca y KREJCAR, Ondrej. A review of infrared thermography for the investigation of building envelopes: Advances and prospects, Energy and Buildings, 2018, vol. 176 , pp. 390-406.

KYLILI, Angeliki; FOKAIDES, Paris A.; CHRISTOU, Petros y KALOGIROU, Soteris A. Infrared thermography (IRT) applications for building diagnostics: A review. Applied Energy, 2014, vol. 134, pp. 531-549.

LUCCHI, Elena. Applications of the infrared thermography in the energy audit of buildings: A review, Renewable and Sustainable Energy Reviews, 2018, vol. 82, n³, pp. 30773090.

MARTÍN OCAÑA, Silvia; CAÑAS GUERRERO, Ignacio y GONZÁLEZ REQUENA, Ignacio.Thermographic survey of two rural buildings in Spain, Energy and Buildings, 2004, vol. 36, pp. 515-523.

MOHR Peter J., TAYLOR Barry N. y NEWELL David B. CODATA Recommended Values of the Fundamental Physical Constants: 2006, Review of Modern Physics, 2008, vol. 80, pp. 715.

NARDI, Inole; LUCCHI, Elena; DE RUBEISA, Tullio y AMBROSINIA, Dario. Quantification of heat energy losses through the building envelope: A state of-the-art, Building and Environment, 2018, vol. 146, pp. 190-205.

PORRAS-AMORES, César; MAZARRÓN, Fernando S. y CAÑAS, Ignacio. Using quantitative infrared thermography to determine indoor air temperature, Energy and Buildings, 2013, vol. 65, pp. 292-298.
ROCHA, J. H. A.; SANTOS, C.F. y PÓVOAS, Y.V. Evaluation of the infrared thremography technique for capillarity moisture detection in buildings, Procedia Structural Integrity, 2018, vol. 11, pp. 107-113.

SCHNEIDER, Lucas. Las 25 recomendaciones de la IEA en políticas públicas sobre eficiencia energética. Observatorio de la Energía, Tecnología e Infraestructura para el Desarrollo [en línea]. 2015, junio. [Consultado 01 noviembre 2018]. Disponible en: http://www.oetec.org/nota.php?id=1258\&area $=7$

TEJEDOR, Beatriz; CASALS, Miguel; GANGOLELLS, Marta y ROCA, Xavier. Quantitative internal infrared thermography for determining in-situ thermal behaviour of façades. Energy and Buildings, 2017, vol. 151, pp. 187-197.

THOUVENEL, Julie. Find a modern and quick method to determine the $U$ value and the thermal characteristics of a building envelope using an IR camera [en línea]. Master of Science Thesis.

$\mathrm{KTH}$ School of Industrial Engineering and Management. Energy Technology. Stockholm, 2012. [Consultado 1 noviembre 2018]. Disponible en: http://kth.diva-portal.org/smash/get/ diva2:603444/FULLTEXT01 